\title{
Negative Integral Powers of a Bidiagonal Matrix
}

\author{
By Gurudas Chatterjee
}

\begin{abstract}
The elements of the inverse of a bidiagonal matrix have been expressed in a convenient form. The higher negative integral powers of the bidiagonal matrix exhibit an interesting property: the $(i j)$ th element of the $(-m)$ th power is equal to the product of the corresponding element of the inverse by a Wronski polynomial, viz., the complete symmetric function of degree $(m-1)$ of the diagonal elements, $d_{i}, d_{i+1}, \ldots, d_{j}$, of the inverse matrix.
\end{abstract}

1. Introduction. Positive integral powers of a bidiagonal matrix with a fixed diagonal element $b$ and superdiagonal element 1, have been reported by Varga [1]. In the present note, we shall find the negative integral powers of a general $n \times n$ bidiagonal matrix $B$, having diagonal elements $b_{i}, i=1,2, \ldots, n$, and superdiagonal elements $c_{j}, j=1,2, \ldots, n-1$.

One may express $B=(I-\Gamma) D^{-1}$, where $I$ is the identity matrix and $D^{-1}$ a diagonal matrix composed of the diagonal elements of $B$. $\Gamma$ is null, except for the elements $\Gamma_{i+1}=-c_{i} / b_{i+1}$, for $i=1,2, \ldots, n-1$, on its first superdiagonal. The powers of $\Gamma$ can easily be evaluated. In fact, the nonzero elements of $\Gamma^{m}$ are given by

$$
\left(\Gamma^{m}\right)_{i+m}=\prod_{k=i}^{i+m-1}\left(-c_{k} / b_{k+1}\right), \quad \text { for } i=1,2, \ldots, n-m,
$$

occurring only on the $m$ th superdiagonal.

The inverse $E_{1}$ of $B$ may be calculated either by the usual method of cofactors, or from the following expansions:

$$
\begin{aligned}
E_{1} & =B^{-1}=D[I-\Gamma]^{-1} \\
& =D\left[I+\Gamma+\Gamma^{2}+\Gamma^{3}+\cdots+\Gamma^{n-1}\right] .
\end{aligned}
$$

The elements of $E_{1}$ may be written in a convenient form as:

$$
\begin{aligned}
e_{1}(i, j) & =0 & & \text { for } i>j, \\
& =1 / b_{j}=d_{j} & & \text { for } i=j, \\
& =d_{i} \prod_{k=i}^{j-1}\left(-c_{k} / b_{k+1}\right) & & \text { for } i<j .
\end{aligned}
$$

The inverse is upper triangular but is not necessarily bidiagonal.

2. Powers of the Inverse. The product of $E_{1}$ with itself is a matrix $E_{2}$, which is also upper triangular. Elements of $E_{2}$ are given by

$$
\begin{aligned}
e_{2}(i, j) & =e_{1}(i, j) \sum_{k=i}^{j}\left[d_{k}\right] & & \text { for } i \leqq j, \\
& =0 & & \text { for } i>j .
\end{aligned}
$$

Received August 6, 1973.

AMS (MOS) subject classifications (1970). Primary 15 A09. 
Result (2) may be generalized. In fact, the $n$th power of $E_{1}$ is an upper triangular matrix $E_{n}$ where the $(i, j)$ th element, for $i \leqq j$, is given by

$$
e_{n}(i, j)=e_{1}(i, j) \sum_{k_{1}=i}^{j} \sum_{k_{2}=i}^{k_{1}} \sum_{k_{3}=i}^{k_{2}} \ldots \sum_{k_{n-2}=i}^{k_{n-3}} \sum_{k_{n-1}=i}^{k_{n-2}}\left[d_{k_{1}} d_{k_{2}} d_{k_{3}} \cdots d_{k_{n-2}} d_{k_{n-1}}\right] .
$$

Proof. Let us assume that result (3) is true for $n=m$.

$$
e_{m+1}(i, j)=\sum_{k_{0}=i}^{j}\left[e_{m}\left(i, k_{0}\right) e_{1}\left(k_{0}, j\right)\right],
$$

the other terms in the summation for $1 \leqq k_{0} \leqq i-1$ and $j+1 \leqq k_{0} \leqq n$, are zero, as both $e_{m}(p, q)$ and $e_{1}(p, q)$ are zero for $p>q$.

By writing the expression for $e_{m}\left(i, k_{0}\right)$ from result $(3)$, which is assumed to be valid for $n=m$, we have

$$
\begin{aligned}
e_{m+1}(i, j)= & \sum_{k_{0}=i}^{j}\left[e_{1}\left(i, k_{0}\right) e_{1}\left(k_{0}, j\right)\right] \\
& \cdot \sum_{k_{1}=i}^{j} \sum_{k_{2}=i}^{k_{1}} \sum_{k_{3}=i}^{k_{2}} \cdots \sum_{k_{m-2}=i}^{k_{m-3}} \sum_{k_{m-1}=i}^{k_{m-2}}\left[d_{k_{1}} d_{k_{2}} d_{k_{3}} \cdots d_{k_{m-2}} d_{k_{m-1}}\right] .
\end{aligned}
$$

The first summation is done by Eq. (2) and the expression reduces to

$$
e_{m+1}(i, j)=e_{1}(i, j) \sum_{k_{0}=i}^{j}\left[d_{k_{0}}\right] \cdot \sum_{k_{1}=i}^{j} \sum_{k_{2}=i}^{k_{1}} \sum_{k_{3}=i}^{k_{2}} \cdots \sum_{k_{m-2}=i}^{k_{m-3}} \sum_{k_{m-1}=i}^{k_{m-2}}\left[d_{k_{1}} d_{k_{2}} d_{k_{3}} \cdots d_{k_{m-2}} d_{k_{m-1}}\right] \text {. }
$$

After grouping the summations together, we find that the result is true for $n=m+1$.

It has already been found true for $n=2$ in Eq. (2), and therefore, by mathematical induction, we have the proof.

3. Thanks are due to the Director of this Institute for his kind permission to publish this paper.

Computer Centre

Central Mechanical Engineering Research Institute

Durgapur-9, West Bengal, India

1. R. S. VARGA, Matrix Iterative Analysis, Prentice-Hall, Englewood Cliffs, N.J., 1962, p. 14. MR 28 \# 1725 . 